\title{
The role of neutrophil-lymphocyte ratio as a prognostic indicator in patients undergoing nephrectomy for renal cell carcinoma
}

\author{
Nathan Grimes ${ }^{1}$; Cathal Hannan ${ }^{2}$; Matthew Tyson ${ }^{3}$; Ali Thwaini ${ }^{4}$ \\ ${ }^{1}$ Forth Valley Royal Hospital, Larbert, Scotland; ${ }^{2}$ Royal Victoria Hospital, Belfast, Northern Ireland; ${ }^{3}$ Craigavon \\ Area Hospital, Craigavon, Northern Ireland; ${ }^{4}$ Belfast City Hospital, Belfast, Northern Ireland
}

Cite as: Can Urol Assoc J 2018 March 19; Epub ahead of print.

http://dx.doi.org/10.5489/cuaj.4872

Published online March 19, 2018

\section{Abstract}

Introduction: Prognosis in patients with cancer is influenced by underlying tumour biology and also the host inflammatory response to the disease. There is limited evidence to suggest that an elevated neutrophil-lymphocyte ratio (NLR) predicts a poorer prognosis in patients undergoing nephrectomy for renal cell carcinoma (RCC). The aim of this paper is to investigate if patients undergoing nephrectomy for RCC with NLR $\leq 4$ have a better overall and recurrence-free survival than patients with NLR $>4$.

Methods: All patients who underwent nephrectomy at a single centre between January 1, 2011 and December 31, 2014 were identified. Patients were included if postoperative histology demonstrated RCC and if preoperative GPS was available. Patients were excluded if nephrectomy was not curative intent (i.e., cytoreductive nephrectomy), if primary tumour was graded to be T3b-4 disease, if there was presence of nodal or metastatic disease on preoperative staging, or if adequate followup notes were not available. Primary and secondary outcomes were overall survival and recurrence-free survival, respectively. Results: A total of 154 patients were included in analysis of overall survival; 146 patients were included in analysis of recurrence-free survival. Patients with NLR $\leq 4$ had a much better overall survival than patients with NLR >4 (95\% vs 78\%; $\mathrm{p}=0.0219$ ). Patients with NLR $>4$ also had higher rates of recurrence ( $\mathrm{p}=0.0218$ ).

Conclusion: NLR may be a useful tool in identifying patients who may benefit from more frequent surveillance in the early postoperative period and may allow clinicians to offer surveillance schemes tailored to the individual patient. 


\section{Introduction}

The incidence of renal cancer has increased over the past thirty five years from 7 per 100,000 to 20 per 100,$000 ;^{1}$ this increase in incidence is expected to continue to 32 per 100,000 by $2035 .{ }^{2}$ Renal cancer currently has the seventh highest incidence of all cancers in the UK. ${ }^{2}$ Renal cell carcinoma (RCC) accounts for approximately $85 \%$ of all renal malignancies. ${ }^{3}$ Over three quarters of patients will have localised disease at time of diagnosis ${ }^{4,5}$ and may be candidates for nephrectomy with curative intent. Despite resection of localised disease, a significant proportion of patients will subsequently develop metastatic disease. This has been reported to occur in $10-30 \%$ patients ${ }^{4,6-8}$ and so the majority of patients undergo long-term post-operative radiological surveillance.

The European Association of Urology currently recommend that surveillance should be based on an individual patient's risk of recurrence, however there is insufficient evidence to recommend any one surveillance scheme to be optimum. ${ }^{9}$ As there is currently no goldstandard surveillance protocol, prognostic indicators based on patient factors may contribute to determining a patient's risk of recurrence and therefore guide intensity of post-operative surveillance.

The neutrophil-lymphocyte ratio (NLR) has been demonstrated to predict prognosis in patients undergoing treatment for a variety of malignancies. ${ }^{10,11}$ The reason that biomarkers that measure for inflammatory processes may be of as a prognostic tool in patients with malignancy is that prognosis for these patients depends, not only on underlying tumour biology, but also the patient's immune response to this disease process. An elevated NLR usually corresponds with a poorer prognosis; this is seen as a reflection of an inflammatory response to the underlying malignant process.

NLR has been investigated as a prognostic indicator in renal cell carcinoma, however the evidence is limited. Several studies investigating the role of NLR in patients undergoing treatment for metastatic RCC demonstrated patients with elevated NLR to have poorer prognosis. ${ }^{3,12}$ The role of NLR in predicting prognosis in patients undergoing nephrectomy for RCC was investigated in a recent review ${ }^{13}$ and identified only four studies. Only two of these studies reported on overall survival but both demonstrated elevated NLR to predict poorer prognosis. ${ }^{14,15}$ Cancer-specific survival and rate of recurrence were also reported but a statistically significant difference was not demonstrated in any study. ${ }^{14-16}$ Many different cutoff values have been described when comparing elevated with non-elevated NLR but there does not appear to be an optimum value.

The aim of this paper is to investigate the role of NLR in predicting overall survival (OS) and recurrence-free survival (RFS) in patients undergoing nephrectomy for localised RCC.

\section{Methods}

A retrospective electronic case note review was conducted on all patients who underwent nephrectomy at a single centre between 01.01.11 and 31.12.14. All patients had full staging imaging pre-operatively in form of CT of chest, abdomen and pelvis and disease was 
recorded in accordance with TNM classification. Patients identified were including in this study if inclusion and exclusion criteria were met.

Inclusion criteria:

- Post-operative histology demonstrated RCC

- Disease was localised (T1-T3a)

- Pre-operative NLR was available: this was based on blood samples taken at a preoperative assessment clinic when patients were well (i.e. no concurrent acute illness). Where blood tests were abnormal and this is thought to be due to a transient acute illness, blood tests were repeated pre-operatively, during same admission as elective surgery.

- Adequate follow-up notes available

Exclusion criteria:

- Nephrectomy was for benign disease

- Malignancy was not RCC

- Nephrectomy was not with curative intent (i.e. cytoreductive nephrectomy)

- Locally advanced disease (T3b-T4)

- Presence of nodal or metastatic disease

- Other significant pathology that would impact survival

Primary outcome studied was overall survival (OS) defined as time from nephrectomy to time of death, or 01.07.16 if death did not occur. Secondary outcome was recurrence-free survival (RFS) defined as time from nephrectomy to radiological diagnosis of recurrence, or most recent radiological investigation excluding recurrence. NLR $\leq 4$ was taken as normal and NLR $>4$ was considered to be elevated. Kaplan-Meier curves were generated for each cohort and OS and RFS were compared using logrank test.

\section{Results}

253 patients were identified during the study period, 99 of whom were excluded for the following reasons:

- 45 patients had benign disease

- 12 patients had malignancy other than RCC

- 19 patients had nodal or metastatic disease

- 7 patients had T3b disease

- 5 patients had significant concurrent pathology

- 11 patients had insufficient data available

154 patients were included and their characteristics are summarised in Table 1. Of the 154 patients included in this study, all had sufficient follow-up data to determine OS, however eight patients had insufficient follow-up data for inclusion in RFS and so 146 patients were included in the analysis of this. OS and RFS were plotted on Kaplan-Meier curves comparing NLR $\leq 4$ with NLR $>4$ in Figure 1 and Figure 2.

Patients with a normal NLR had a better OS than patients with NLR $>4$. While OS was similar in the first two years following surgery, the survival curves diverged after this, 
with 3-year OS being $95 \%$ and $78 \%$ for patients with NLR $\leq 4$ and NLR $>4$ respectively $(\mathrm{p}=0.0219)$.

An NLR $>4$ also predicted a poorer RFS, particularly in the early post-operative period compared with patients with NLR $\leq 4$ ( $\mathrm{p}=0.0218$ ). 1-, 2- and 3-year RFS for NLR $\leq 4$ versus NLR $>4$ was $97 \%$ versus $88 \%$, $95 \%$ versus $78 \%$ and $92 \%$ versus $78 \%$. This difference in RFS lessoned by the fourth post-operative year; $82 \%$ for NLR $\leq 4$ versus $78 \%$ for NLR $>4$.

\section{Discussion}

These results support that an elevated NLR is associated with poorer OS and RFS in patients with RCC undergoing nephrectomy with curative intent. Within the first two post-operative years, OS was comparable between patients with NLR $\leq 4$ and NLR $>4$, however the survival curves diverged significantly after this point. Interestingly, the greatest difference in RFS between the two cohorts was within three years, with a smaller difference at four years after resection. This poor RFS in the early stage following resection may explain the difference in OS after three years as this may correspond with the time period that patients who had developed early recurrence had died from their disease. An NLR $>4$ may be a useful indicator for which patients may be suitable for more intensive post-operative surveillance in the first few years following resection so that should there be recurrent disease, it can be recognised at the earliest possible stage and be considered for treatment.

There is no one particular cut-off value when considering NLR and this makes comparing studies in this subject difficult as there is significant variation in reported literature. Pichler et al. ${ }^{15}$ investigated the role of NLR in patients undergoing nephrectomy for clear-cell RCC (NLR $<3.3 n=398 ;$ NLR $\geq 3.3 n=280)$ and demonstrated that a cut-off of 3.3 showed a statistically significant difference in OS, but not cancer-specific survival or metastasis-free survival. Viers et al. ${ }^{14}$ also compared outcomes in patients undergoing nephrectomy for clear-cell RCC using 4 as a cut-off and found that there was a statistically significant difference in OS and cancer-specific survival, but not metastasis-free survival. Other studies have investigated the prognostic value of NLR without assigning a particular cut-off representing elevated or non-elevated NLR, but have noted that patients who develop metastatic disease within five years of nephrectomy tend to have a higher pre-operative NLR than those who develop metastases greater than five years after nephrectomy. ${ }^{17}$ In this study, a cut-off of 4 was used as this has been demonstrated to be useful as a prognostic tool in various different settings, including prognosis of patients being treated for localised RCC, patients with metastatic RCC, ${ }^{18}$ and in other malignancies. ${ }^{19}$

Another issue which makes comparing studies on this subject difficult is patient inclusion. Previously published papers include patients with T1-T4 disease, ${ }^{15}$ and other included patients with nodal disease. ${ }^{14}$ This study only included patients with localised disease, and this was defined as T1-T3aN0M0. Patients with T3b-T4 disease were excluded as they would require surgery more extensive than radical nephrectomy (some may require significant vascular surgery) and should these patients have been included, their outcomes may have skewed the results of their cohort. Patients with nodal disease were also excluded on the basis that the possible role for NLR may be in guiding intensity of post-operative 
follow-up. Patients who have positive nodes on histology will already be undergoing more intense follow-up as they would be expected to have a higher risk of recurrent disease, regardless of what their pre-operative NLR may have been, and so NLR is not relevant for this group of patients.

While this study highlights the possible benefit of NLR as a prognostic tool, it also demonstrates that it would not be of use as a diagnostic tool in distinguishing benign from malignant disease. Over $80 \%$ of the patients included in this study had a normal NLR. While the use of pre-operative NLR as a predictor of final histology was not investigated in this study, with such a high proportion of patients with malignant disease having a normal ratio, it would be of any diagnostic use in the investigation of a patient with a renal lesion.

One limitation of this study was that it was a retrospective analysis of patients who had already underwent nephrectomy. Another limitation was the duration of follow-up after nephrectomy. Results were not available past five years following nephrectomy.

Furthermore, with patients being included until 31.12.14 and final follow-up data being collected on 01.07.16, a significant number of patients included in this study will only have had a short period of post-operative follow-up, and therefore these patients do not have robust long-term follow-up outcome data. While this study suggests there is a poorer OS and PRS survival associated with NLR $>4$, a longer follow-up period would be required to investigate if this trend is maintained in the long-term.

The results of this study support that an elevated pre-operative NLR is associated with poorer prognosis in patients undergoing nephrectomy for RCC with curative intent. These results are in keeping with current available evidence. Pre-operative NLR may be a useful tool in identifying patients at higher risk of early recurrence following nephrectomy and as such, may be used to identify patients who may benefit from more frequent post-operative surveillance. 


\section{References}

1. Cancer Research UK. Kidney cancer incidence statistics. Available at: http://www.cancerresearchuk.org/health-professional/cancer-statistics/statistics-bycancer-type/kidney-cancer/incidence\#heading-Two. Accessed 19.01.17

2. Cancer Research UK. Kidney cancer statistics. Available at: http://www.cancerresearchuk.org/health-professional/cancer-statistics/statistics-bycancer-type/kidney-cancer\#heading-Zero. Accessed 19.01.17

3. Dirican A, Kucukzeybek Y, Somali I, et al. The association of hematologic parameters on the prognosis of patients with metastatic renal cell carcinoma. J BUON 2013 AprJun;18(2):413-9.

4. Bukowski RM. Prognostic factors for survival in metastatic renal cell carcinoma: update 2008. Cancer 2009 May 15;115(10 Suppl):2273-81.

5. Jagdev SP, Gregory W, Vasudev NS, et al. Improving the accuracy of pre-operative survival prediction in renal cell carcinoma with C-reactive protein. Br J Cancer 2010 Nov 23;103(11):1649-56.

6. Zisman A, Pantuck AJ, Wieder J, et al. Risk group assessment and clinical outcome algorithm to predict the natural history of patients with surgically resected renal cell carcinoma. J Clin Oncol 2002 Dec 1;20(23):4559-66.

7. Park YH, Baik KD, Lee YJ, et al. Late recurrence of renal cell carcinoma $>5$ years after surgery: clinicopathological characteristics and prognosis. BJU Int 2012 Dec;110(11 Pt B):E553-8.

8. Kim SP, Weight CJ, Leibovich BC, et al. Outcomes and clinicopathologic variables associated with late recurrence after nephrectomy for localized renal cell carcinoma. Urology 2011 Nov;78(5):1101-6.

9. European Association of Urology. Renal Cell Carcinoma. Available at: http://uroweb.org/guideline/renal-cell-carcinoma. Accessed 19.01.17

10. Ishizuka M, Nagata H, Takagi K, et al. Combination of platelet count and neutrophil to lymphocyte ratio is a useful predictor of postoperative survival in patients with colorectal cancer. Br J Cancer 2013 Jul 23;109(2):401-7.

11. Yamanaka T, Matsumoto S, Teramukai S, et al. The baseline ratio of neutrophils to lymphocytes is associated with patient prognosis in advanced gastric cancer. Oncology 2007;73(3-4):215-20.

12. Dirican A, Kucukzeybek Y, Erten C, et al. Prognostic and predictive value of hematologic parameters in patients with metastatic renal cell carcinoma: second line sunitinib treatment following IFN-alpha. Asian Pac J Cancer Prev 2013;14(3):2101-5.

13. Grimes N, Tyson M, Hannan C, et al. A systematic review of the prognostic role of hematologic scoring systems in patients with renal cell carcinoma undergoing nephrectomy with curative intent. Clin Genitourin Cancer 2016 Aug;14(4):271-6.

14. Viers BR, Houston Thompson R, et al. Preoperative neutrophil-lymphocyte ratio predicts death among patients with localized clear cell renal carcinoma undergoing nephrectomy. Urol Oncol 2014 Nov;32(8):1277-84. 
15. Pichler M, Hutterer GC, Stoeckigt C, et al. Validation of the pre-treatment neutrophillymphocyte ratio as a prognostic factor in a large European cohort of renal cell carcinoma patients. Br J Cancer 2013 Mar 5;108(4):901-7.

16. De Martino M, Pantuck AJ, Hofbauer S, et al. Prognostic impact of preoperative neutrophil-to-lymphocyte ratio in localized nonclear cell renal cell carcinoma. J Urol 2013 Dec;190(6):1999-2004.

17. Sejima T, Iwamoto H, Morizane S, et al. The significant immunological characteristics of peripheral blood neutrophil-to-lymphocyte ratio and Fas ligand expression incidence in nephrectomized tumor in late recurrence from renal cell carcinoma. Urol Oncol 2013 Oct;31(7):1343-9.

18. Baum Y, Patil D, Huang J, et al. Elevated preoperative neutrophil-to-lymphocyte ratio may be associated with decreased overall survival in patients with metastatic clear cell renal cell carcinoma undergoing cytoreductive nephrectomy. Asian J Urol 2016 Jan;3(1):20-5.

19. Bhatti I, Peacock O, Lloyd G, et al. Preoperative hematologic markers as independent predictors of prognosis in resected pancreatic ductal adenocarcinoma: neutrollymphocyte versus platelet-lymphocyte ratio. Am J Surg 2010;200:197-203 


\section{Figures and Tables}

Fig. 1. Kaplan Meier curve for overall survival following nephrectomy. NLR: neutrophillymphocyte ratio.

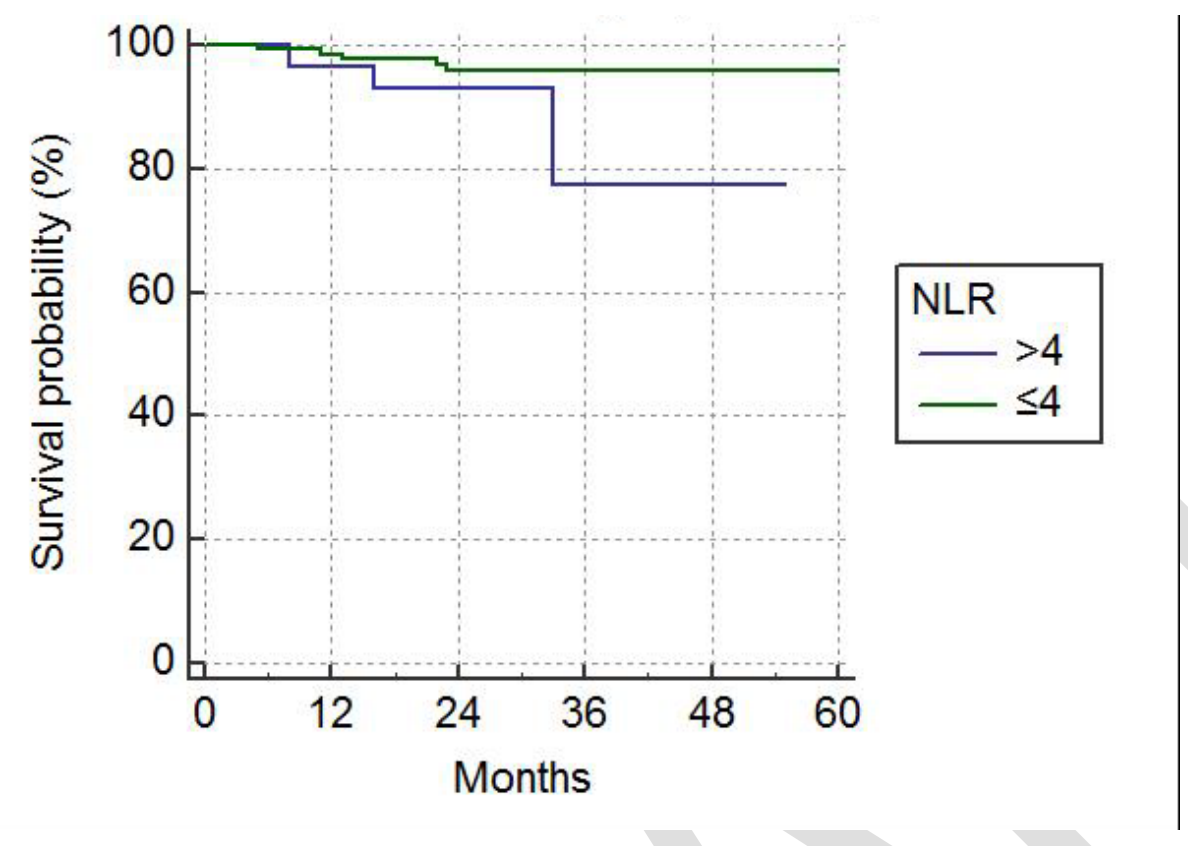

Fig. 2. Kaplan Meier curve for recurrence-free survival following nephrectomy. NLR: neutrophil-lymphocyte ratio.

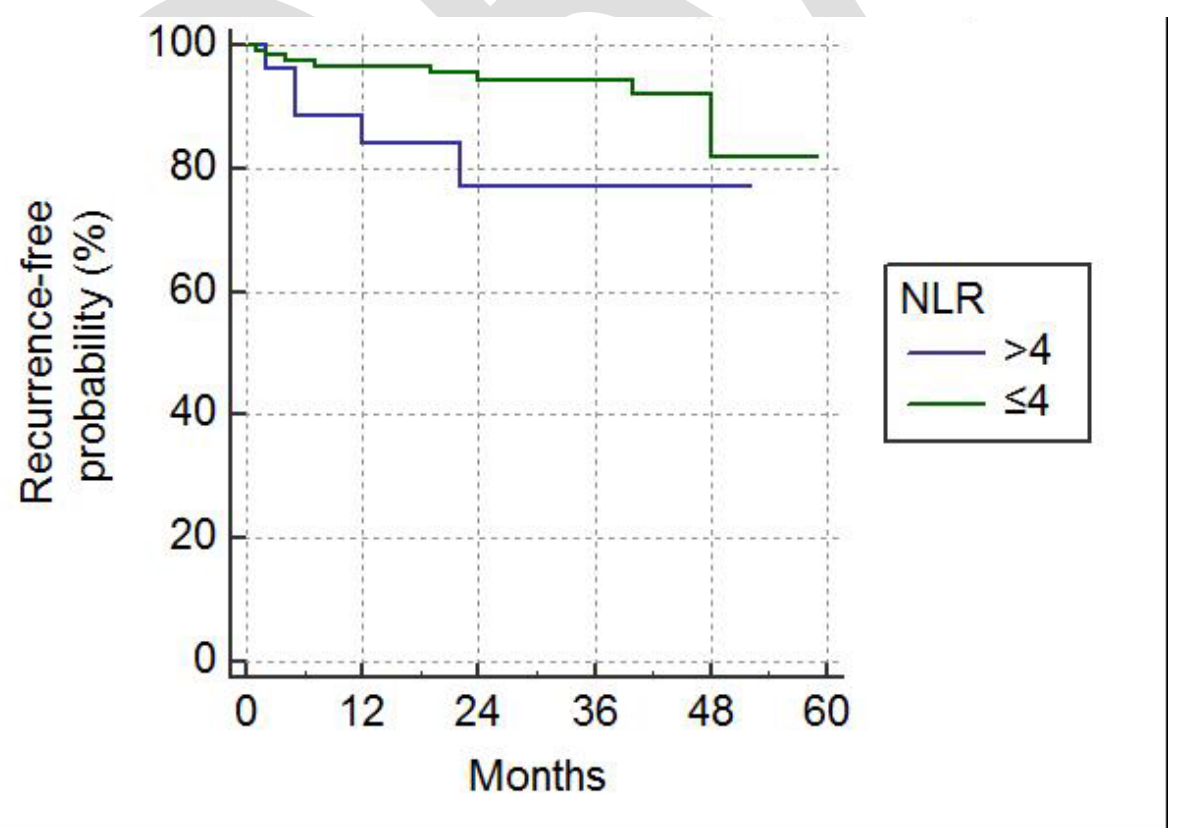




\begin{tabular}{|l|c|c|}
\hline Table 1. Summary of patients included in study \\
\hline Number & NLR $\leq \mathbf{4}$ & NLR $>\mathbf{4}$ \\
\hline Male/female & 126 & 28 \\
\hline Median age (range) & $61 / 65$ & $20 / 8$ \\
\hline T1/T2/T3a & $64.5(26-86)$ & $65(34-88)$ \\
\hline Partial/radical & $87 / 14 / 25$ & $14 / 4 / 10$ \\
\hline
\end{tabular}

NLR: neutrophil-lymphocyte ratio. 